\title{
ÓBITOS PERINATAIS INVESTIGADOS E FALHAS NA ASSISTÊNCIA HOSPITALAR AO PARTOa
}

Perinatal deaths investigated and failures in hospital care delivery

Muertes perinatales investigadas y los fracasos en la atención hospitalaria al parto

\section{RESUMO}

Objetivou-se analisar as falhas na assistência hospitalar ao parto, o perfil materno e as características do feto e do recém-nascido que evoluíram para óbito perinatal, em Belo Horizonte. Métodos: Estudo transversal, dos óbitos perinatais investigados pelo Comitê de Prevenção de Óbitos de Belo Horizonte, ocorridos entre 2003 e 2007. A fonte dos dados foram as fichas de investigação do Comitê. Os dados foram analisados pela distribuição de frequência das variáveis e análise bivariada utilizando-se o teste de Qui-quadrado de Pearson, considerando o nível de significância de 5\% $(p<0,05)$. Resultados: Foram estudados 253 óbitos, a maioria de nascidos a termo, com peso adequado ao nascer. Em 65,6\% dos casos houve falhas na assistência, principalmente relacionadas ao acompanhamento da gestante durante o trabalho de parto e parto. Conclusões: Foram identificados um alto percentual de falhas assistenciais no processo do parto e um grande potencial de evitabilidade dos óbitos perinatais.

Palavras-chave: Mortalidade perinatal. Parto. Assistência hospitalar. Sistemas de informação. Enfermagem;

\begin{abstract}
To analyze the shortcomings in hospital care delivery, the maternal profile and characteristics of the fetus and newborn that died perinatally, in Belo Horizonte. Methods: Cross sectional study of perinatal deaths investigated by the Committee for the Prevention of Deaths of Belo Horizonte, which occurred between 2003 and 2007. The data source was the Committee's investigation forms. Data were analyzed by frequency distribution of variables and the bivariate analysis used the chi-square test, considering the significance level of $5 \%(p<0.05)$. Results: We studied 253 deaths, most born at term with adequate weight infants. In $65.6 \%$ of cases there were gaps in care, mainly related to the monitoring of pregnant women during labor and delivery. Conclusions: We identified a high percentage of failures in the process of care delivery and a great potential avoidability of perinatal deaths.
\end{abstract}

Keywords: Perinatal mortality. Parturition. Hospital Care. Information systems. Nursing.

\section{Resumen}

Analizar las deficiencias en la prestación de la atención hospitalaria al parto, el perfil materno y las características del feto y del recién nacido que murió durante el período perinatal, en Belo Horizonte (MG). Métodos: eEstudio transversal de las muertes perinatales investigadas por el Comité para la Prevención de Muertes de Belo Horizonte, que se produjo entre 2003 y 2007. La fuente de datos fueron informes de investigación del Comité. Los datos fueron analizados mediante distribución de frecuencias de las variables y el análisis bivariado se utilizó la prueba de Qui-Quadrado, teniendo en cuenta el nivel de significación del $5 \%(p<0,05)$. Resultados: se estudiaron 253 muertes, la mayoría de nacidos con peso adecuado. En el $65,6 \%$ de los casos hubo deslices en la atención, principalmente relacionados con la monitorización de mujeres embarazadas durante el trabajo de parto. Conclusiones: se identificó un alto porcentaje de fracasos en el proceso de prestación de atención y un gran potencial de prevención de las muertes perinatales.

Palabras clave: Mortalidad Perinatal. Parto. Atención hospitalaria. Sistemas de información. Enfermería

\footnotetext{
1- Enfermeira, Doutora e Professora Adjunta do Departamento de Enfermagem Materno-Infantil e Saúde Pública - Escola de Enfermagem da Universidade Federal de Minas Gerais. Belo Horizonte-MG. Brasil. E-mail: eufram@enf.ufmg.br; ${ }^{2}$ Enfermeira, Doutora e Professora Associada do Departamento de Enfermagem Materno-Infantil e Saúde Pública - Escola de Enfermagem da Universidade Federal de Minas Gerais. Belo Horizonte-MG. Brasil. E-mail: ednarez@enf.ufmg.br; ${ }^{3}$ Enfermeiro, Doutor e Professor Associado do Departamento de Enfermagem Materno-Infantil e Saúde Pública -

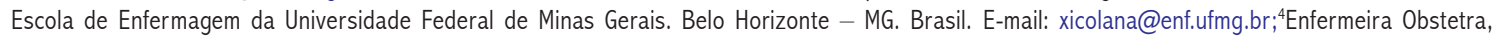
Doutora e Professora Adjunta do Departamento de Enfermagem Materno-Infantil e Saúde Pública - Escola de Enfermagem da Universidade Federal de Minas Gerais. Belo Horizonte - MG. Brasil. E-mail: kleydeventura@uol.com.br
} 


\section{INTRODUÇÃO}

A mortalidade perinatal é um evento raro nos países desenvolvidos, mas comum e ainda pouco valorizado nos países menos desenvolvidos, nos quais se concentra a quase totalidade dessas mortes ${ }^{1}$. Atualmente esses óbitos têm adquirido destaque devido a maior participação do componente neonatal precoce na mortalidade infantil ${ }^{1}$, como também pela disponibilidade de intervenções efetivas de custobenefício comprovadas e capazes de impactar positivamente na redução dessas mortes².

A taxa de mortalidade perinatal tem sido apontada como um indicador sensível da saúde maternoinfantil por refletir aspectos relativos à saúde reprodutiva, ao acesso e à qualidade de recursos disponíveis para assistência ao pré-natal, ao parto e ao neonato ${ }^{1,3}$. No Brasil, a situação da mortalidade perinatal não é conhecida de forma sistemática para todo o país, devido à elevada subnotificação de óbitos fetais e à qualidade insatisfatória da informação disponível sobre a duração da gestação na Declaração de Óbito 4 .

No entanto, experiências pioneiras para o enfrentamento desse problema têm sido implementadas em Belo Horizonte, Minas Gerais, desde o final da década de 1990. Na análise da mortalidade e da assistência perinatal foram identificadas deficiências na estrutura, no processo de atendimento e na organização da rede assistencial as quais se relacionaram às mortes perinatais potencialmente evitáveis. Diante dessa realidade, houve a recomendação de se avançar na qualidade e na responsabilização dos serviços e do sistema de saúde ${ }^{5}$.

Uma das estratégias adotada foi, no ano de 1999, a estruturação da Comissão Perinatal no município, um fórum interinstitucional responsável pela gestão das políticas de atenção à gestante e ao recémnascido na cidade. Vinculado a esta Comissão foi implantado, em 2002, o Comitê de Prevenção do Óbito Fetal e Infantil, com a função de investigar os óbitos, avaliar as circunstâncias de sua ocorrência e apontar diretrizes para intervenções ${ }^{3}$. As investigações realizadas pelo Comitê apresentam informações com potencial para elucidar os níveis ainda elevados da mortalidade perinatal no município, considerando a capacidade instalada de sua rede de serviços de saúde para 0 atendimento à gestante e ao recém-nascido.

Para a melhoria dos indicadores perinatais, as ações dos serviços de saúde devem desenvolver-se de forma integrada e contínua durante a gestação, par to e pós-parto. Particularmente, no processo de assistência ao parto e nascimento, é essencial o cuidado qualificado para o reconhecimento de complicações e adoção de condutas adequadas, além da atenção de qualidade ao recém-nascido, logo após o parto².

Diante do contexto apresentado, conhecer os problemas relacionados à assistência dispensada pelas maternidades às parturientes e neonatos poderá contribuir para a elaboração de medidas preventivas, visando a redução dos óbitos potencialmente evitáveis. Assim, o objetivo deste estudo foi analisar as falhas na assistência hospitalar ao parto, o perfil materno e as características do feto e do recém-nascido que evoluíram para óbito perinatal, em Belo Horizonte.

\section{MÉTODO}

Estudo transversal sobre óbitos perinatais investigados pelo Comitê de Prevenção de Óbitos da Secretaria Municipal de Saúde de Belo Horizonte, Minas Gerais, ocorridos no período de 2003 a 2007. Foram excluídos do estudo os óbitos perinatais domiciliares e os hospitalares de fetos com batimento cardíaco negativo à admissão na maternidade.

Utilizaram-se dados secundários provenientes do Sistema de Informação sobre Mortalidade (SIM) e das investigações realizadas pelo Comitê. A investigação consistiu em uma avaliação detalhada da assistência ao pré-natal, ao parto e ao recém-nascido, com base nos prontuários dos serviços de saúde nos quais a gestante foi atendida e na entrevista domiciliar, com a puérpera ou familiar. Foram critérios para a investigação o óbito de fetos e recém-nascidos filhos de mães residentes em Belo Horizonte, com peso ao nascer igual ou superior a $1.500 \mathrm{~g}$ e ausência de malformação congênita grave ou letal.

Para este estudo, foi elaborado um instrumento específico para a coleta dos dados nos formulários de investigação do Comitê e construído um banco de dados no software Epi Info 10.0. Posteriormente esse banco de dados foi concatenado ao banco do SIM para acrescentar a variável causa básica da morte. As variáveis incluídas foram relativas ao óbito (tipo de óbito, causa básica da morte e classificação de evitabilidade), ao feto e ao recém-nascido (peso ao nascer, idade gestacional, tipo de parto), à gestante (idade, escolaridade e condição materna na gestação) e às falhas na assistência hospitalar ao processo de parto e nascimento. A condição materna foi considerada desfavorável quando presente alguma patologia ou vulnerabilidade social.

As falhas na assistência foram identificadas na conclusão da investigação do caso pelo Comitê, baseando-se em evidências científicas preconizadas nos protocolos do município e Ministério da Saúde ${ }^{6}$. Foram 
identificadas quatro categorias de falhas assistenciais, relativas à admissão da gestante na maternidade (avaliação incompleta e demora no atendimento); ao acompanhamento da gestante durante o trabalho de parto e parto (intervalo dos controles maternofetal superior ao recomendado, diagnóstico tardio das distocias do trabalho de parto e demora no manejo das complicações do trabalho de parto e parto); à relação profissional-cliente insatisfatória (atendimento desumanizado e falta de informação); e à assistência imediata ao recém-nascido na sala de parto (falta de profissional para assistir o RN e demora em iniciar a reanimação neonatal). 0 acesso da gestante à maternidade, apesar de não se constituir uma falha hospitalar, foi também identificada.

A classificação de análise de evitabilidade de óbitos adotada foi a de Wigglesworth ${ }^{7}$, por sua correlação com a qualidade da atenção perinatal. Esta classificação estratifica os óbitos por peso ao nascer e os classifica em cinco subgrupos de causas: morte anteparto, malformação congênita, imaturidade, asfixia e outras causas específicas ${ }^{7}$. As causas básicas de morte foram agrupadas segundo a lista reduzida de tabulação das causas de óbitos neonatais, proposta por França e Lansky $(2009)^{8}$, a qual agrupa as causas de morte de forma a direcionar as ações preventivas de saúde.

Os dados foram analisados pela distribuição de frequências das variáveis, e foi realizada a análise bivariada para estimar a associação entre a existência de falhas na assistência hospitalar ao parto e as variáveis relativas à parturiente, ao feto e ao recémnascido. Utilizou-se o teste de Qui-quadrado de Pearson, e foi considerado o nível de significância de $5 \%(p<0,05)$. Foi utilizado o software Epi info versão 10.0 para esta análise.

Este estudo foi aprovado nos Comitês de Ética da Universidade Federal de Minas Gerais, parecer No. 242/ 08, e da Secretaria Municipal de Saúde de Belo Horizonte, parecer No. 042/2008. Foram respeitados os aspectos éticos de acordo com a Resolução 196/96 do Conselho Nacional de Saúde.

\section{RESULTADOS}

No período foram registrados 2.710 óbitos perinatais no município. A população desse estudo foi constituída pelos 253 casos investigados, ocorridos após a admissão da gestante no hospital. As falhas na assistência ocorreram em mais da metade desses óbitos (Figura1).

Figura 1. Óbitos perinatais investigados. Belo Horizonte, 2003 a 2007.

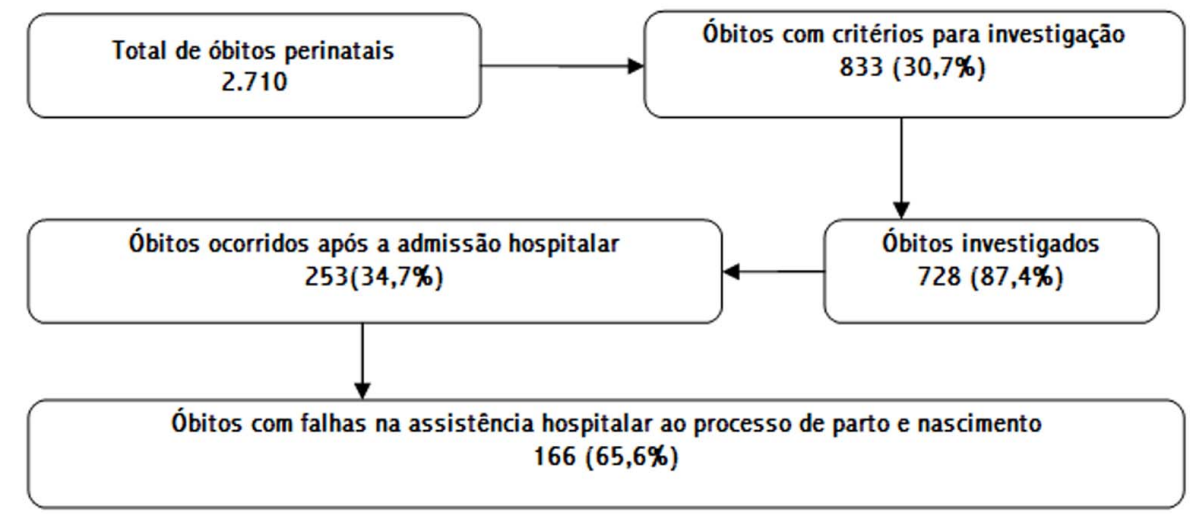

Fonte: Sistema de Informação de Mortalidade e Comitê de Prevenção de Óbitos BH-Vida. Secretaria Municipal de Saúde de Belo Horizonte

As categorias de falhas identificadas no processo de assistência ao parto e nascimento na maternidade são apresentadas na Tabela 1 . $\mathrm{Na}$ admissão da gestante à maternidade houve falhas em $22,3 \%$ dos casos, com destaque para a avaliação incompleta. 0 acompanhamento da gestante durante 0 
trabalho de parto e parto foi a categoria com mais falhas registradas $(91,6 \%)$, destacando-se a frequência do intervalo de avaliação materno-fetal superior ao recomendado e a demora no manejo das complicações do trabalho de parto e parto. A relação profissional/cliente insatisfatória, com queixas de atendimento desumanizado e falta de informação, foi identificada em $11,5 \%$ dos casos. As falhas relativas à assistência imediata ao recém-nascido na sala de parto foram identificadas em menor escala, com destaque para a demora em iniciar a reanimação neonatal. Em cerca de 15,0\% dos casos, além das falhas assistências, houve dificuldade de acesso da gestante à maternidade.

Tabela.1. Categorias de falhas na assistência ao processo de parto e nascimento identificadas na investigação do óbito perinatal. Belo Horizonte, 2003 a 2007.

\begin{tabular}{|c|c|c|}
\hline Categorias de Falhas & $\mathbf{N}$ & $* \%$ \\
\hline \multicolumn{3}{|l|}{ Admissão da gestante } \\
\hline Avaliação incompleta & 27 & 16,3 \\
\hline Demora no atendimento & 10 & 6,0 \\
\hline Subtotal & 37 & 22,3 \\
\hline \multicolumn{3}{|l|}{ Acompanhamento da gestante durante o trabalho de parto e parto } \\
\hline Intervalo dos controles matemo-fetal superior ao recomendado & 73 & 44,0 \\
\hline Diagnóstico tardio das distocias do trabalho de parto & 25 & 15,0 \\
\hline Demora no manejo das complicações do trabalho de parto e parto & 54 & 32,6 \\
\hline Subtotal & 152 & 91,6 \\
\hline \multicolumn{3}{|l|}{ Relação profissional-cliente insatisfatória } \\
\hline Atendimento desumanizado & 13 & 7,8 \\
\hline Falta de informação & 06 & 3,6 \\
\hline Subtotal & 19 & 11,4 \\
\hline \multicolumn{3}{|l|}{ Assistência imediata ao RN na sala de parto } \\
\hline Demora em iniciar a reanimação neonatal & 07 & 4,2 \\
\hline Falta de profissional para assistira o recém-nascido & 02 & 1,2 \\
\hline Subtotal & 09 & 5,4 \\
\hline Dificuldade de acesso da gestante à maternidade & 24 & 14,5 \\
\hline Total de falhas & 241 & 100,0 \\
\hline
\end{tabular}

* Percentual calculado em relação ao total de 166 casos com falhas.

Fonte: Comitê de Prevenção de Óbitos BH-Vida. Secretaria Municipal de Saúde de Belo Horizonte.

A Tabela 2 apresenta as falhas na assistência em relação às características do óbito, do feto, do neonato e das gestantes. Prevaleceram os óbitos de fetos e recém-nascidos com peso ao nascer superior a $2.500 \mathrm{~g}$, nascidos após 36 semanas de idade gestacional, de parto vaginal, de mães de 20 a 34 anos de idade e escolaridade de oito anos e mais. Na maioria dos casos foi detectada condição materna desfavorável na gestação, pela presença de doenças hipertensivas, infecções do trato urinário e vulnerabilidades sociais. 0 maior percentual de falhas na assistência ao parto e nascimento ocorreu para o óbito fetal, nas gestações com duração igual ou superior a 37 semanas e diante de condição materna favorável. As variáveis tipo de óbito, duração da gestação e condição materna associaram-se de forma significativa às falhas na assistência $(p<0,05)$.

Nos casos com falhas na assistência hospitalar, a principal causa básica de morte, segundo a CID-10, foi a asfixia/hipóxia, seguida das demais causas, destacando-se, nesse grupo, as mortes fetais de causa indeterminada (Figura 2). A classificação de evitabilidade de Wigglesworth apontou que mais da metade dos óbitos foram por asfixia e 13,0\%, por morte anteparto (Figura 3). 
Óbitos perinatais investigados

Martins EF, Rezende EM, Lana FCF, Souza KV

Tabela 2. Características das parturientes, do feto e do recém-nascido segundo falhas na assistência hospitalar ao parto e nascimento. Belo Horizonte, 2003 a 2007.

\begin{tabular}{|c|c|c|c|c|}
\hline \multirow[t]{2}{*}{ Variável } & \multicolumn{2}{|c|}{ Falhas na assistência } & \multirow[t]{2}{*}{ Total de óbitos } & \multirow[t]{2}{*}{ p-valor ${ }^{2}$} \\
\hline & Sim & Não & & \\
\hline Tipo de óbito & $N(\%)$ & $N(\%)$ & $N(\%)$ & $<0,05$ \\
\hline Fetal & $72(84,71)$ & $13(15,29)$ & $85(33,60)$ & \\
\hline Neonatal precoce & $94(55,95)$ & $74(44,05)$ & $168(66,40)$ & \\
\hline Total & $166(65,6)$ & $87(34,5)$ & $253(100,0)$ & \\
\hline Duraçāo da gestaçāo (semanas) & & & & $<0,05$ \\
\hline$\leq 36$ & $48(53,93)$ & $41(46,07)$ & $89(39,04)$ & \\
\hline 37 a 41 & $102(73,38)$ & $37(26,62)$ & $139(60,96)$ & \\
\hline Ignorado & $16(9,63)$ & $9(10,34)$ & $25(9,88)$ & \\
\hline Peso ao nascer (gramas) & & & & $>0,05$ \\
\hline 1.500 a 2.499 & $52(58,43)$ & $37(41,57)$ & $89(35,17)$ & \\
\hline 2.500 e mais & $114(69,51)$ & $50(30,49)$ & $164(64,83)$ & \\
\hline Tipo de parto & & & & $>0,05$ \\
\hline Vaginal & $86(72,27)$ & $33(27,73)$ & $119(52,89)$ & \\
\hline Cesárea & $64(60,38)$ & $42(39,62)$ & $106(47,11)$ & \\
\hline Ignorado & $16(9,6)$ & $12(13,8)$ & $28(11,06)$ & \\
\hline Idade matema (anos) & & & & $>0,05$ \\
\hline$<20$ & $22(73,33)$ & $8(26,67)$ & $30(13,10)$ & \\
\hline 20 a 34 & $106(63,86)$ & $60(36,14)$ & $166(72,49)$ & \\
\hline 35 e mais & $20(60,61)$ & $13(39,39)$ & $33(14,41)$ & \\
\hline Ignorado & $18(10,8)$ & $6(6,8)$ & $24(9,5)$ & \\
\hline Escolaridade materna (anos) & & & & $>0,05$ \\
\hline Até 7 & $54(65,85)$ & $28(34,15)$ & $82(38,32)$ & \\
\hline 8 e mais & $88(66,67)$ & $44(33,33)$ & $132(61,68)$ & \\
\hline Ignorado & $24(14,5)$ & $15(17,2)$ & $39(15,4)$ & \\
\hline Condiçāo materna desfavorável & & & & $<0,05$ \\
\hline Sim & $128(62,44)$ & $77(37,56)$ & $205(84,71)$ & \\
\hline Não & $30(81,08)$ & $7(18,92)$ & $37(15,29)$ & \\
\hline Ignorado & $8(4,8)$ & $3(3,4)$ & $11(4,4)$ & \\
\hline
\end{tabular}

Teste de Qui-quadrado de Pearson.

Fonte: Sistema de Informação de Mortalidade e Comitê de Prevenção do óbito fetal e infantil de Belo Horizonte. Secretaria Municipal de Saúde de Belo Horizonte.

Figura 2. Distribuição dos óbitos perinatais investigados com falhas na assistência hospitalar segundo agrupamentos de causas básicas de morte. Belo Horizonte, 2003 a 2007.

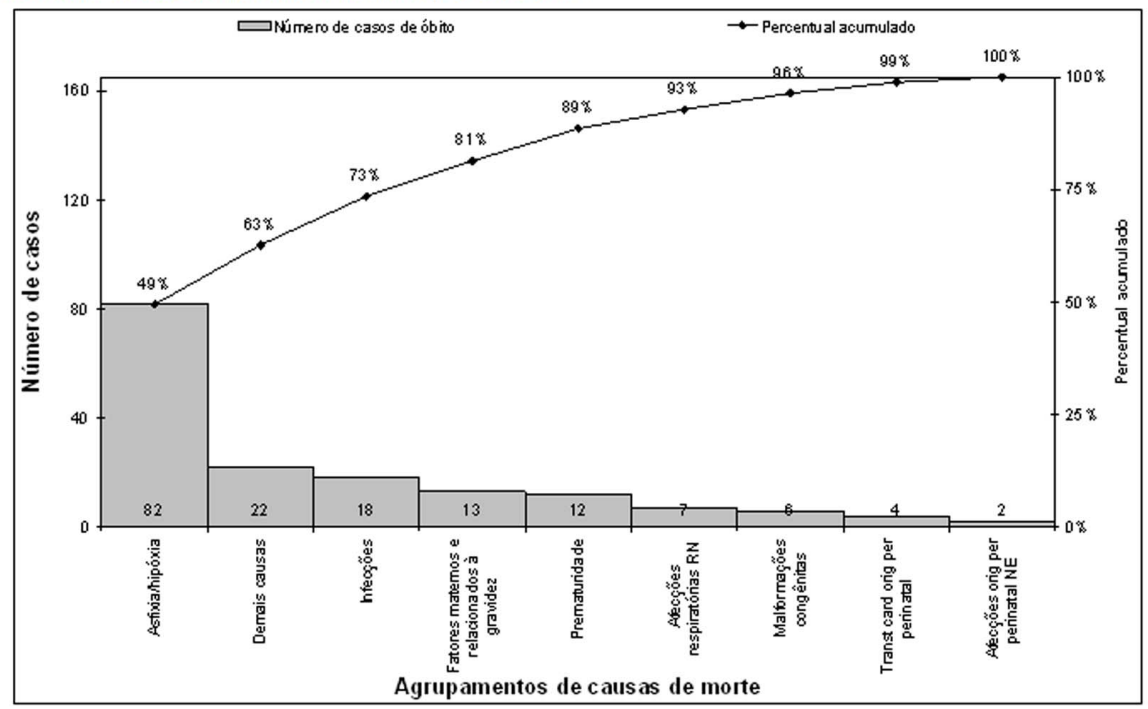

Fonte: Comitê de Prevenção de Óbitos BH-Vida. Secretaria Municipal de Saúde da Prefeitura de Belo Horizonte. 
Figura 3. Distribuição dos óbitos perinatais investigados com falhas na atenção hospitalar segundo Classificação de Evitabilidade de Wingglesworth. Belo Horizonte, 2003 a 2007.

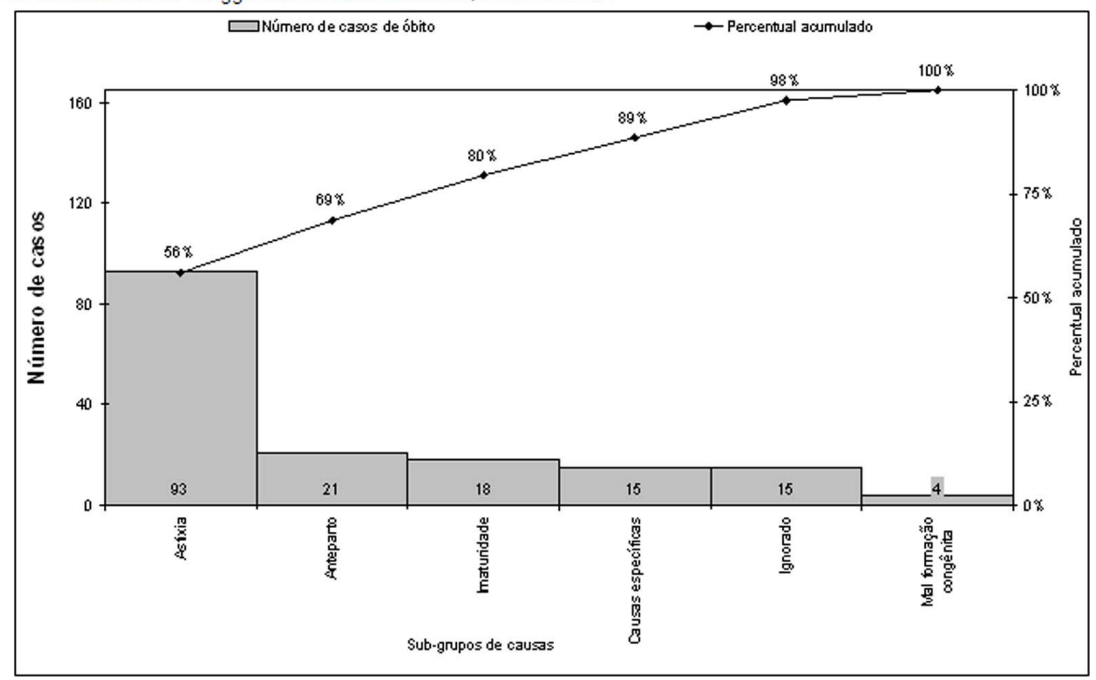

Fonte: Comitê de Prevenção de Óbitos BH-Vida. Secretaria Municipal de Saúde de Belo Horizonte.

\section{DISCUSSÃO}

A predominância neste estudo de óbitos de fetos e neonatos a termo e com peso adequado ao nascer difere do perfil clássico de mortalidade perinatal descrito na literatura ${ }^{1}$, em que prevalece o baixo peso ao nascer e a prematuridade, principais determinantes da mortalidade perinatal ${ }^{1}$. Essa diferença ocorreu por se tratar de óbitos com critérios de investigação previamente definidos. Esses óbitos, em fetos e crianças viáveis, são aparentemente de mais fácil prevenção, e sua redução deve ser alvo de intervenções para o alcance de melhores indicadores perinatais no município.

0 elevado percentual de falhas identificado no processo de assistência hospitalar ao parto e nascimento em Belo Horizonte aponta que melhorias devem ser implantadas nestes serviços, desde a admissão da gestante na maternidade até 0 atendimento ao recém-nascido na sala de parto. A presença de falhas na admissão da gestante na maternidade, como constatado em Belo Horizonte, também foi identificada em um estudo realizado em São Paulo, e culminou em parto domiciliar não planejado com perdas perinatais ${ }^{9}$. Para melhorar 0 atendimento na chegada da gestante às maternidades foi elaborado, pela Comissão Perinatal da SMSA/PBH, em conjunto com todas as maternidades da rede SUS do município, um protocolo para o Acolhimento com Classificação de Risco em Obstetrícia. Esse protocolo prioriza os atendimentos segundo a gravidade, o risco e a vulnerabilidade de cada usuária ${ }^{10}$. Espera-se que essa modalidade de atendimento contribua para estabelecer uma escuta mais efetiva desde a admissão, com a valorização das queixas da mulher e o entendimento de suas vulnerabilidades, visando condutas especíicas de acordo com as necessidades identificadas.

$\mathrm{Na}$ assistência durante o trabalho de parto e parto observou-se, em relação a outro estudo realizado em Belo Horizonte na década anterior ${ }^{5}$, redução da frequência do intervalo de avaliação materno-fetal superior ao recomendado. Entretanto, esta falha não devia existir, visto que o adequado acompanhamento da gestante e do feto, durante o trabalho de parto e parto, é uma função básica dos serviços de atenção obstétrica ${ }^{11} .0$ adequado controle materno e fetal durante 0 trabalho de parto permite identificar situações de complicações e adotar as condutas apropriadas em tempo oportuno. Assim sendo, é importante avaliar os fatores que dificultam a adoção de práticas assistenciais benéficas e eficazes na prevenção de resultados perinatais adversos. Podem ser necessárias mudanças no processo de trabalho, capacitação da equipe e assistência multidisciplinar na perspectiva de trabalho colaborativo.

A presença de falhas assistenciais nas maternidades pode ser também decorrente do modelo predominante de atenção obstétrica no país, modelo hospitalocêntrico e com excesso de intervenções que podem levar a danos iatrogênicos. A discussão de mudanças desse modelo tem sido foco das políticas públicas atuais no país, com destaque para a recente criação da Rede Cegonha que, no âmbito do Sistema Único da Saúde (SUS), propõe uma rede de cuidados voltada à assistência de qualidade e humanizada à mulher e à criança ${ }^{12}$. Outro aspecto importante para superar os problemas na assistência perinatal é o cumprimento da regulação vigente, que prevê acolhimento, ambiência, humanização da atenção e da gestão, além de infraestrutura física, recursos humanos, equipamentos e materiais necessários à operacionalização do serviço, de acordo com a demanda e modalidade de assistência prestada ${ }^{11}$.

As queixas de atendimento desumanizado e falta de informação foram falhas constatadas em menor escala nesse estudo. Isso possivelmente reflete o desconhecimento das mulheres sobre seus direitos, como também o fato de esse aspecto não ter sido foco específico das investigações, embora relevante para a qualidade do atendimento. Um estudo realizado no Sul do Brasil, com mulheres que tiveram um filho 
com asfixia perinatal grave, também apontou aspectos negativos vivenciados na assistência recebida no processo de parturição. Foram destacados a exposição a situações desumanas, o não respeito ao direito de ter um acompanhante durante o processo e o despreparo do profissional para assistir as intercorrências ${ }^{13}$.

A presença de falhas na assistência ao recém-nascido na sala de parto, especialmente a demora para iniciar a reanimação neonatal, foi uma situação identificada em alguns casos estudados, o que pode acarretar em graves danos ao recém-nascido. Essa situação, provavelmente, está mais relacionada com a atuação dos recursos humanos do que com a falta de equipamentos, conforme resultados de um estudo ${ }^{14}$ realizado em salas de parto de hospitais públicos das capitais brasileiras, incluindo Belo Horizonte. Na ocasião foi identificado que, em sua maioria, as maternidades tinham infraestrutura adequada para a reanimação neonatal, entretanto, havia a necessidade de treinamento dos profissionais para realizarem efetivamente o procedimento. Esta conduta realizada de forma efetiva, quando necessária, contribui para reduzir a morbimortalidade neonatal, sobretudo por asfixia, ainda elevada no Brasil ${ }^{14}$.

0 tipo de parto não se associou à ocorrência de falhas na assistência hospitalar entre os óbitos investigados de Belo Horizonte. Esse resultado foi similar ao de outro estudo que evidenciou que a mortalidade perinatal está mais relacionada com a qualidade da assistência obstétrica e neonatal do que com a via de parto ${ }^{15}$. Em relação às variáveis que se associaram às falhas na assistência obstétrica hospitalar (tipo de óbito, idade gestacional e condição materna desfavorável), faz-se necessário aprofundar esta análise, com a avaliação de outros fatores mais diretamente relacionados ao processo da assistência. A maior incidência de falhas entre os óbitos fetais pode ser explicada pelo diagnóstico e manejo tardios das distocias do trabalho de par to e parto. 0 trabalho de parto de fetos prematuros pode ter sido um fator protetor para as falhas assistenciais, em virtude de essa situação ser considerada de alto risco, o que alerta para uma atenção mais rigorosa.

A condição materna desfavorável na gestação foi muito frequente entre os óbitos investigados em Belo Horizonte, situação também encontrada em um estudo realizado no estado do Paraná com óbitos infantis investigados ${ }^{16}$. Em ambos os estudos, os agravos maternos mais comuns foram as doenças hipertensivas e a infecção do trato urinário. Essas complicações na gravidez associam-se ao aumento da morbidade e mortalidade fetal e neonatal ${ }^{5}$, mas podem ser prevenidos com uma assistência pré-natal de qualidade, em que se realizam 0 diagnóstico precoce e o tratamento adequado.

0 percentual mais elevado de mortes relacionadas à hipóxia intrauterina e à asfixia ao nascer, em relação ao encontrado em outros estudos ${ }^{17-18}$, justifica-se por se referir a óbitos cujos critérios para investigação excluem outras causas, como a prematuridade extrema e as malformaç̃̃es congênitas graves. As mortes por hipóxia e asfixia devem ser vistas como um problema no município, especialmente porque os nascimentos ocorrem em maternidades, diferentemente dos países menos desenvolvidos onde a maioria dessas mortes é decorrente de partos domiciliares, por falta de acesso ao serviço ${ }^{18}$. As mortes intraparto decorrentes da asfixia é um indicador sensível da qualidade da assistência durante 0 trabalho de parto e nascimento ${ }^{1}$. Portanto, esforços devem ser envidados para avanços na assistência obstétrica em Belo Horizonte, com vistas a reduzir a mortalidade e também as sequelas decorrentes da asfixia intraparto.

A classificação de evitabilidade de Wigglesworth destacou, além da asfixia, o agrupamento das mortes anteparto. Esses óbitos geralmente se relacionam com as complicações da gravidez e doenças maternas de mais difíicil prevenção, além de falhas na atenção pré-natal e de muitos casos de morte inexplicada ${ }^{1}$. A morte fetal de causa não especificada também foi relevante no estudo. Pode ser resultante de má nutrição fetal, aberrações cromossômicas e infecções não diagnosticadas no pré-natal, e mais de uma condição pode contribuir para esses óbitos ${ }^{17}$. Assim sendo, é importante recorrer às investigações desses óbitos para o esclarecimento de suas circunstâncias, o que possibilita, em alguns casos, esclarecer a causa da morte e corrigir a causa básica no SIM.

A inclusão no estudo de apenas óbitos investigados traz algumas limitações, como a identificação de um maior potencial de evitabilidade e de falhas assistenciais na população estudada. Aponta, entretanto, que o foco das ações dos serviços de saúde deve ser direcionado para a redução dos óbitos mais preveníveis. A coleta dos dados em fonte secundária e de forma retrospectiva limitou a abordagem de alguns aspectos relativos à assistência, como a adoção de práticas que favorecem a fisiologia do nascimento nos serviços e uso do partograma, pela incompletude dos dados e inexistência da variável durante todo o período das investigações. Porém teve como vantagens a agilidade e 0 baixo custo, pelo uso da informação já disponível no Comitê de Prevenção de Óbitos.

\section{CONSIDERAÇÕES FINAIS}

Este estudo aponta um perfil de óbitos perinatais com alto potencial de evitabilidade, em sua maioria, de fetos e recém-nascidos a termo e peso adequado ao nascer, condições favoráveis à viabilidade fetal e neonatal. Predominam as mortes por asfixia evidenciadas tanto pela classificação de evitabilidade de Wigglesworth quanto pela causa básica de morte. Identificase alto percentual de falhas assistenciais, especialmente relacionadas à admissão da gestante na maternidade e ao seu acompanhamento durante $o$ trabalho de parto e parto.

0 estudo a partir da investigação de óbitos considerados eventos sentinelas permite identificar aspectos que devem ser enfrentados pelos serviços de saúde, para a promoção de um 
nascimento seguro e a prevenção de mortes evitáveis. Nesse sentido, a Comissão Perinatal de Belo Horizonte vem desenvolvendo ações para a qualificação da assistência nas maternidades e o trabalho em rede. Estas ações posteriormente devem ser avaliadas quanto ao possível impacto na melhoria da atenção nas maternidades e na redução da mortalidade perinatal no municíio.

Enfim, para melhorar a assistência e os indicadores perinatais, é preciso haver investimentos direcionados para os serviços de saúde, a formação profissional e a informação das mulheres e da sociedade como um todo, sobre a existência de tecnologia e de direitos que favorecem um nascimento mais seguro e humanizado. 0 trabalho deve ser desenvolvido de forma integrada e contínua, incluindo planejamento e ações do setor saúde e intersetoriais.

\section{REFERÊNCIAS}

1. World Health Organization, Department of Making Pregnancy Safer. Neonatal and perinatal mortality: country, regional and global estimates. Geneva(SUI): World Health Report; 2006.

2. Bhuta ZA, Darmstadt GL, Hasan BS, Haws RA. Community-based interventions for improving perinatal and neonatal health outcomes in developing countries: a review of the evidence. Pediatrics. 2005 Feb; 115(2 Suppl): 519-617.

3. Lansky S. Gestão da qualidade e da integralidade do cuidado em saúde para a mulher e a criança no SUS-BH: a experiência da comissão perinatal. Divulg. saúde debate. 2006 ago; (36): 10-17.

4. Rede Interagencial de Informações para Saúde-RIPSA (BR), Organização Pan-Americana da Saúde (OPAS). Indicadores básicos para saúde no Brasil: conceitos e aplicações. $2^{0}$ ed. Brasília(DF): RIPSA; 2008.

5. Lansky S, Franca E, César CC, Monteiro Neto LC, Leal MC. Perinatal deaths and childbirth healthcare evaluation in maternity hospitals of the Brazilian Unified Health System in Belo Horizonte, Minas Gerais, Brazil, 1999. Cad. saúde pública. 2006 jan; 22 (1): 117-30.

6. Ministério da Saúde (BR), Secretaria de Vigilância em Saúde, Secretaria de Atenção à Saúde. Manual de vigilância do óbito infantil e fetal e do Comitê de Prevenção do Óbito Infantil e Fetal. $2^{\circ}$ ed. Brasilia(DF): Ministério da Saúde; 2009.

7. Wiggleswortth, J. S. Monitoring perinatal mortality: a pathophysiological approach. Lancet. 1980 set. 27; 2(8196): 684-6

8. França E, Lansky S. Mortalidade infantil neonatal no Brasil: situação, tendências e perspectivas. In: Rede Interagencial de Informações para Saúde-Ripsa. Demografia e Saúde: contribuição para análise de situação e tendências. Brasília(DF): Ministério da Saúde; 2009. p. 83-112.

9. Almeida MF, et al. Partos domiciliares acidentais na região sul do Município de São Paulo. Rev. saúde pública. 2005 jun; 39(3): 366-75.
10. Belo Horizonte(MG). Secretaria Municipal de Saúde. Comissão Perinatal. Associação Mineira de Ginecologia e Obstetricia. Protocolo do acolhimento com classificação de risco em obstetrícia e principais urgências obstétricas. Belo Horizonte(MG): [Secretaria Municipal de Saúde]; 2010.

11. Agência Nacional de Vigilância Sanitária (BR). Diretoria Colegiada. Resolução n. ${ }^{\circ}$ 36, de 3 de junho de 2008. Dispõe sobre regulamento técnico para funcionamento dos serviços de atenção obstétrica e neonatal. Diário Oficial República Federativa do Brasil, Brasília(DF), 9 jul. 2008; (130): 45.

12. Ministério da Saúde (BR). Portaria nº 1.459, de 24 de junho de 2011. Institui, no âmbito do Sistema Único de Saúde - SUS - a Rede Cegonha. Diário Oficial República Federativa do Brasil, 27 jun. 2011; Seção 1:109.

13. Milbrath VM, Amestoy SC, Soares DC, Siqueira HCH. Vivencias maternas sobre a assistência recebida no processo de parturição. Esc Anna Nery. 2010 jul/set; 14(2): 462-7.

14. Almeida MFB, Guinsburg R, Costa JO, Anchieta LM, Freire LMS. Material and human resources for neonatal resuscitation in public maternity hospitals in Brazilian state capitals. São Paulo med. j. 2008 may; 126(3): 156-60.

15. Duarte, G, Coltro PS, Bedone RV, Nogueira AA, Gelonezzi GM, Franco $\sqcup$. Trends in the modes of delivery and their impact on perinatal mortality rates. Rev. saúde pública. 2004 jun; 38(3): 379-84.

16. Santana IP, Santos JM, Costa JR, Oliveira RR, Orlandi MHF, Mathias TAF. Aspects of infant mortality, according to an investigation of death. Acta paul. enferm. 2011; 24(4): 556-62.

17. Confidential Enquiry into Maternal and Child Health (CEMACH). Perinatal Mortality 2006: England, Wales and Northern Ireland. London(ENG): CEMACH; 2008.

18. Lawn JE, Lee A CC, Kinney M, Sibley L, Carlo W, Paul VK, Pattinson R, Darmstadt GL. Two million intrapartum-related stillbirths and neonatal deaths: Where, why, and what can be done? Int I Gynaecol Obstet. 2009 Oct;107 Suppl 1:S5-18, S19.

\section{NOTA}

aArtigo extraído da tese "Mortalidade perinatal e avaliação da assistência ao pré-natal, ao parto e ao recém-nascido, Belo Horizonte, Minas Gerais", apresentada ao programa de pós-graduação da Escola de Enfermagem, Universidade Federal de Minas Gerais, 2010. 